\title{
Crystal Structure of Sodium Guaiazulene Sulfonate Hemihydrate
}

\author{
Kazuko Kohara,* Hohjun TaChibana,* Izumi Funae,* Makoto Kamezawa,* \\ Takehiko OHTANI, ${ }^{*}$ Kazuo Eda, ${ }^{* * \dagger}$ and Kimiaki YAMAMURA** \\ *Konan Chemical Industry Co. Ltd., 5-21 Nakagawa, Takatsuki, Osaka 569-0066, Japan \\ **Department of Chemistry, Faculty of Science, Kobe University, 1-1 Rokkodai, Nada-ku, \\ Kobe 657-8501, Japan
}

\begin{abstract}
The crystal structure of sodium guaiazulene sulfonate hemihydrate was determined by X-ray diffraction analysis. The compound crystallizes in the monoclinic space group $P 2_{1} / c$ with four molecules per unit cell. The cell parameters are $a=$ 23.271(4) $\mathrm{A}, b=7.1965(12) \AA, c=8.8334(15) \AA, \beta=93.178(3)^{\circ}, V=1477.0(4) \AA^{3}$. The structure was refined to the final $R 1=0.092$. It reveals that the compound has the layer structure, stacked with hydrophobic interactions at one side and Coulomb interactions at the other side.
\end{abstract}

(Received November 28, 2005; Accepted January 23, 2006; Published on web March 27, 2006)

Guaiazulene, 7-isopropyl-1,4-dimethylazulene, (GA) is an active component of the plant, "Matricaria chamomilla L.", which has been used as self-medication for gastritis or canker sores for many years. Sodium guaiazulene sulfonate $\left(\mathrm{GASO}_{3} \mathrm{Na}\right)$ is a hydrophilic derivative of $\mathrm{GA}$, and has excellent anti-inflammatory and wound-healing properties. Thus, $\mathrm{GASO}_{3} \mathrm{Na}$ has been widely used as an essential component of

Table 1 Crystal and experimental data

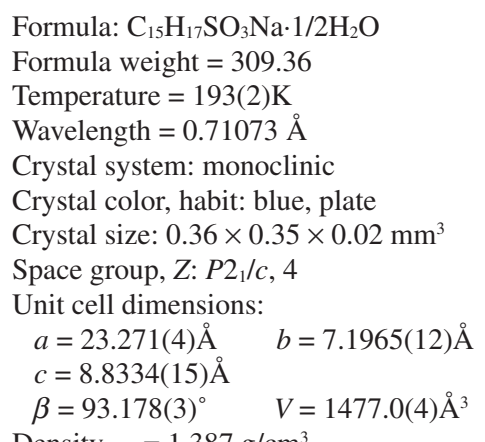

Density calc. $=1.387 \mathrm{~g} / \mathrm{cm}^{3}$

No. of reflections used $=7832$

$\theta_{\max }=27.38^{\circ}$

$R 1[2436$ refs. $I>2 \sigma(I)]=0.092$

$w R 2$ (all data) $=0.250$

$(\Delta / \sigma)_{\max }=0.000$

$(\Delta \rho)_{\max }=0.616 \mathrm{e}^{-3}$

$(\Delta \rho)_{\min }=-0.769 \mathrm{e}^{-3}$

Measurement: Bruker smart 1000

Program system: SHELX

Structure determination: direct method

Refinements: full-matrix least-squares on $F^{2}$

Crystallographic data have been deposited at the CCDC, 12 Union Road, Cambridge CB2 1EZ, UK and copies can be obtained on request, free from charge, by quoting the publication citation and deposition number: CCDC290058.

To whom correspondence should be addressed.

E-mail: eda@kobe-u.ac.jp pharmaceuticals and cosmetics. However, it is comparatively unstable and gradually decomposes in the solid state, even at room temperature. Some ways to combat this unwanted behavior have been tried. Antioxidants and weak basic metallic salts or alkaline-earth metals were employed as stabilizing agents by Kohlstaedt et al., ${ }^{1}$ while an amino acid was added by Kohara et al. ${ }^{2}$ Kawamata et al..$^{3,4}$ proposed stabilized amorphous $\mathrm{GASO}_{3} \mathrm{Na}$ prepared by spray-drying or freeze-drying aqueous solutions of $\mathrm{GASO}_{3} \mathrm{Na}$ containing a polymeric excipient, such as polyvinyl pyrrolidone. In order to discuss the stabilization methods, it is useful to know the crystal structure of $\mathrm{GASO}_{3} \mathrm{Na}$. It has, however, not been known up to now. We report here on the crystal structure of the title compound, which is one of the $\mathrm{GASO}_{3} \mathrm{Na}$ forms usually used for pharmaceuticals and<smiles>Cc1cc([SH](=O)([O-])[O-])c2c(C)ccc([14CH3])cc1-2</smiles>

Fig. 1 Chemical structure of the title compound.

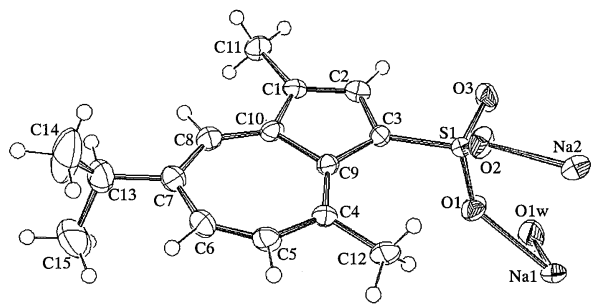

Fig. 2 Perspective view of the molecular structure of the title compound. The thermal ellipsoids are drawn at the 50\% probability level. The spheres present the hydrogen atoms. (See text for the hydrogen atoms of the hydration water $(\mathrm{O} 1 \mathrm{w})$ and the two $\mathrm{Na}^{+}$cation sites.) 
Table 2 Atomic coordinates and equivalent isotropic displacement parameters $\left(\AA^{2}\right)$

\begin{tabular}{lrrrr}
\hline Atom & \multicolumn{1}{c}{$x$} & \multicolumn{1}{c}{$z$} & \multicolumn{1}{c}{$U_{\text {eq }}$} \\
\hline C1 & $0.2615(2)$ & $0.4510(7)$ & $-0.2202(6)$ & $0.0243(11)$ \\
C2 & $0.3212(2)$ & $0.4489(7)$ & $-0.2120(6)$ & $0.0253(11)$ \\
C3 & $0.3424(2)$ & $0.4844(7)$ & $-0.0623(5)$ & $0.0208(10)$ \\
C4 & $0.2936(2)$ & $0.5450(7)$ & $0.1873(5)$ & $0.0234(10)$ \\
C5 & $0.2433(2)$ & $0.5817(8)$ & $0.2648(6)$ & $0.0309(12)$ \\
C6 & $0.1862(2)$ & $0.5854(9)$ & $0.2226(6)$ & $0.0364(13)$ \\
C7 & $0.1562(2)$ & $0.5466(9)$ & $0.0819(6)$ & $0.0328(12)$ \\
C8 & $0.1847(2)$ & $0.5054(8)$ & $-0.0487(6)$ & $0.0309(12)$ \\
C9 & $0.2948(2)$ & $0.5113(7)$ & $0.0313(6)$ & $0.0222(10)$ \\
C10 & $0.2422(2)$ & $0.4898(7)$ & $-0.0765(6)$ & $0.0257(11)$ \\
C11 & $0.2240(2)$ & $0.4210(9)$ & $-0.3613(6)$ & $0.0346(13)$ \\
C12 & $0.3479(3)$ & $0.5440(9)$ & $0.2882(6)$ & $0.0349(13)$ \\
C13 & $0.0913(3)$ & $0.5500(12)$ & $0.0749(8)$ & $0.0508(18)$ \\
C14 & $0.0676(3)$ & $0.7405(16)$ & $0.1135(11)$ & $0.085(3)$ \\
C15 & $0.0666(4)$ & $0.3941(19)$ & $0.1705(11)$ & $0.091(4)$ \\
S1 & $0.41724(5)$ & $0.49518(16)$ & $-0.02159(13)$ & $0.0196(3)$ \\
O1 & $0.43304(16)$ & $0.3361(5)$ & $0.0730(5)$ & $0.0338(9)$ \\
O2 & $0.43029(16)$ & $0.6696(5)$ & $0.0545(5)$ & $0.0341(9)$ \\
O3 & $0.44208(16)$ & $0.4876(6)$ & $-0.1693(4)$ & $0.0382(11)$ \\
Na1* & $0.51163(17)$ & $0.1979(6)$ & $0.1961(4)$ & $0.0263(9)$ \\
Na2* & $0.50648(17)$ & $0.8033(6)$ & $0.1926(4)$ & $0.0266(9)$ \\
O1w & 0.5 & 0.0 & 0.0 & $0.070(3)$ \\
& & & & \\
\hline
\end{tabular}

*0.50 occupancy for $\mathrm{Na} 1$ and $\mathrm{Na} 2$.

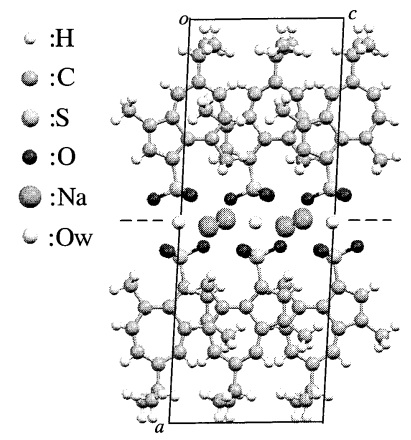

Fig. 3 Ball-and-stick views of the crystal structure of the title compound along the $b$-axis. The broken line represents the plane on which the $\mathrm{Na}^{+}$cations are roughly located.

cosmetics. $^{5}$

Single crystals of the title compound were prepared by recrystallization from a methanol solution of $\mathrm{GASO}_{3} \mathrm{Na}$. Blue crystals were obtained from the solution at room temperature by slow evaporation for a few days. Diffraction data were collected by a Bruker smart $1000 \mathrm{CCD}$ diffractometer at $193 \mathrm{~K}$ with graphite-monochromated Mo- $K_{\alpha}$ radiation $(\lambda=0.71073$ $\AA$ ). The structure was solved by direct methods. ${ }^{6}$ The nonhydrogen atoms were refined anisotropically by a full-matrix least-squares method. ${ }^{7}$ The hydrogen atoms of the hydration water, located on a center of symmetry, were not used in the present refinement. The positions of other hydrogen atoms were calculated geometrically. SHELX programs were used for these calculations.
Table 3 Selected bond distances and angles

\begin{tabular}{llll}
\hline \multicolumn{2}{c}{ Distances $(\AA)$} & \multicolumn{2}{c}{ Angles $\left(^{\circ}\right)$} \\
\hline S1-O1 & $1.453(4)$ & O1-S1-O2 & $112.1(2)$ \\
S1-O2 & $1.448(4)$ & O1-S1-O3 & $112.7(3)$ \\
S1-O3 & $1.458(4)$ & O2-S1-O3 & $111.4(3)$ \\
\hline
\end{tabular}

The crystal and experimental data are given in Table 1. This compound crystallizes in the monoclinic $P 2_{1} / c$ space group. The chemical structure, the molecular structure, and the final atomic parameters of non-hydrogen atoms are shown in Figs. 1 and 2, and Table 2 respectively. The crystal structure reveals that $\mathrm{GASO}_{3}{ }^{-}$anions stack themselves up, forming lamellar layers with a one-molecule width (Fig. 3). In the layers the aromatic rings are almost parallel to each other, but staggered so as not to completely overlap. Thus, the methyl group at the 1position is located just over the adjacent seven-membered rings. Hydrophobic isopropyl and hydrophilic sulfo groups are placed separately, so that isopropyl groups are projecting at one side of the layer, while the sulfo groups are at the other side. Then, the layers are stacked with hydrophobic interactions among isopropyl groups at one side and Coulomb interactions between sulfo groups and $\mathrm{Na}^{+}$cations at the other side, alternating upside down and rightside up, along the $a$-axis. A couple of stacked layers correspond to the repetition sequence of the crystal. The $\mathrm{Na}^{+}$cation is disordered at two sites with a half occupancy. Thus, the $\mathrm{Na}^{+}$cations at the two sites are drawn just as a dumbbell in Fig. 3. The $\mathrm{Na}^{+}$cation is formally coordinated to six oxygen atoms (four from two adjacent sulfo groups and two from two adjacent hydration water molecules). The hydration water molecule is located almost on the Na plane (see Fig. 3) and coordinates to four adjacent $\mathrm{Na}^{+}$cations. The sulfo groups from the upper and lower layers face each other at the interface of the two layers, and are related by a pseudo-rotation-reflection axis, $S_{6}$. All of the $\mathrm{S}-\mathrm{O}$ bond distances and all of the O-S-O bond angles are $c a .1 .45 \AA$ and $c a .112^{\circ}$, respectively (Table 3 ). Thus, the three oxygen atoms of the sulfo group are described as being almost equivalent.

\section{References}

1. E. Kohlstaedt and W. Schmidt, Germany Patent, 1958, 1034327.

2. K. Kohara and H. Tachibana, Japan Patent, 1974, S4911219.

3. M. Kawamata, K. Ushimaru, H. Gohshi, and H. Miyasako, Japan Patent, 1989, H01-50225.

4. M. Kawamata, K. Ushimaru, H. Gohshi, and H. Miyasako, Japan Patent, 1989, H01-50226.

5. K. Nakamichi, T. Nakano, H. Yasuura, S. Izumi, and Y. Kawashima, Eur. J. Pharm. Biopharm., 2003, 56, 347.

6. G. M. Sheldrick, Acta Cryst., 1990, A46, 467.

7. G. M. Sheldrick, SHELXL-97, Program for the Refinement of Crystal Structures, University of Gottingen Germany, 1997. 